Statistica Neerlandica (2007) Vol. 61, nr. 1, pp. 61-74

\title{
Heuristic estimates in shortest path algorithms
}

\author{
Wim Pijls \\ Econometric Institute, Erasmus University Rotterdam, P.O. Box 1738, \\ 3000 DR Rotterdam, The Netherlands
}

\begin{abstract}
Shortest path problems occupy an important position in operations research as well as in artificial intelligence. In this paper we study shortest path algorithms that exploit heuristic estimates. The wellknown algorithms are put into one framework. Besides, we present an interesting application of binary numbers in the shortest path theory.
\end{abstract}

Keywords and Phrases: network flows, graph theory, operations research, search problems.

\section{Introduction}

Finding a shortest path in a network is a major topic in operations research (OR) and combinatorial optimization. However, shortest path algorithms exploiting heuristic estimates are hardly studied in the literature on those areas. On the other hand, the artificial intelligence literature (AI) addresses search problems involving heuristic estimates. The related algorithms can be viewed as shortest path algorithms. Only in the past few years, a connection between the two disciplines has been established in the study of real road networks. In this paper, algorithms from both fields are combined into one framework. As a bonus, a surprising relationship between binary numbers and shortest path algorithms is presented. Furthermore, a general termination proof for shortest path algorithms is given. A notable role is played by the so-called invariants, a common concept in computer science. An invariant is a property holding after each iteration during the execution of an algorithm. Invariants are proved by induction on the number of iterations. We only discuss the induction step, while proving invariants.

\subsection{Preliminaries}

A graph or network $G$ is defined by a pair $(V, E)$ with $V$ the set of nodes and $E$ the set of edges. A path is a sequence of nodes without duplicate elements. In the graph one node $s$ is designated as the start or the source. The shortest path and its

pijls@few.eur.nl

Most of the topics addressed in this paper have been extensively discussed by Antoon and myself, before and after the publication of my thesis.

(C) 2007 The Author. Journal compilation (C) 2007 VVS.

Published by Blackwell Publishing, 9600 Garsington Road, Oxford OX4 2DQ, UK and 350 Main Street, Malden, MA 02148, USA. 
length from $s$ to any other node $v$ is looked for. The weight or length of an edge $(u, v)$ is denoted by $d(u, v)$, whereas $\hat{d}(u, v)$ denotes the length of the shortest path from $u$ to $v$.

\subsection{Overview}

Section 2 recalls some basics of the shortest path theory. This section contains a new termination proof. Section 3 puts Dijkstra and related algorithms, well known in $\mathrm{OR}$, on the one hand, and $\mathrm{A}^{*}$ and the search algorithms, mostly treated in the AI literature, on the other hand into one framework. Although the algorithms are not new, the idea of an underlying framework is. Furthermore, a new property of so-called proper estimates is given. Section 4 presents a relationship between binary numbers and a shortest path instance. The study of paths in real road networks is briefly discussed in section 5 . Section 6 discusses the search space visited by shortest path algorithms.

\section{The shortest path problem}

The problem of finding the shortest path length between a source $s$ and a target $t$ can be described as an linear programming (LP) problem (see Papadimitriou and Steiglitz, 1982). The dual of this LP problem is:

$$
\begin{aligned}
& \max g(t), \\
\text { s.t. } & g(v)-g(u) \leq d(u, v), \quad \forall u, v \in V \\
& g(s)=0 .
\end{aligned}
$$

We assume that the graph does not contain a cycle of negative length, because finding a shortest path in a graph with negative cycles is NP-hard. This is proved in GAREY and Johnson (1979).

\subsection{The generic algorithm}

The foregoing LP description leads us in a natural way to the generic algorithm (see Algorithm 1).

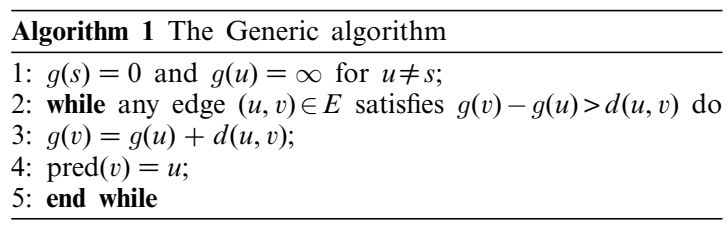

The values $g(v)$ and pred $(v)$ are called the label and the predecessor of $v$ respectively. The operation in line 3 is called edge relaxation in some studies (see, e.g. (C) 2007 The Author. Journal compilation (c) 2007 VVS. 
Goodrich and TAMassia, 2002). The edge was stretched out by the difference of $g(v)$ and $g(u)$ and by the new value $g(v)$ it is relaxed and comes back to its resting state. Given the generic algorithm, the following question arises: does this algorithm terminate for every instance? In most of the literature, an affirmative answer is assumed. However, we did not find clear evidence for this answer. Here, we give a termination proof. The key to this proof is provided by the following invariant.

Invariant 1. For any $w \in V, g(w)$ is associated with a path $P=\left(s=p_{0}, p_{1}, \ldots, p_{n-1}\right.$, $\left.p_{n}=w\right)$ such that

$$
g(w)=\sum_{i=0}^{n-1} d\left(p_{i}, p_{i+1}\right)
$$

(so $g(w)$ equals the length of $P$ ) and

$$
g\left(p_{k}\right) \leq \sum_{i=0}^{k-1} d\left(p_{i}, p_{i+1}\right) \quad \text { for } 1 \leq k<n .
$$

Proof. Suppose an edge $(u, v)$ is selected. If $v \neq p_{k}, 1 \leq k \leq n$, the invariant is maintained trivially for $w$. If $v=p_{k}, 1 \leq k<n, g(w)$ and its associated path are unaffected, but $g\left(p_{k}\right)$ takes a smaller value. The relation

$$
g\left(p_{k}\right) \leq \sum_{i=0}^{k-1} d\left(p_{i}, p_{i+1}\right)
$$

and hence the invariant are maintained. If $v=p_{n}=w$, a new path will be associated with $w$ : the path associated with $u$ is enhanced with edge $(u, v)$. Moreover, in this case, the invariant is preserved for $w$ and trivially for the other nodes. Suppose an edge $(w, v)$ is selected. We show that $v \neq p_{k}$ for any $p_{k}, 0 \leq k \leq n$, in the path associated with $w$. If $v=p_{k}$ was the case, we would have before the update of $v=p_{k}$ :

$$
\begin{aligned}
g\left(p_{k}\right)=g(v)>g(w)+d(w, v) & =\sum_{i=0}^{k-1} d\left(p_{i}, p_{i+1}\right)+\sum_{i=k}^{n-1} d\left(p_{i}, p_{i+1}\right)+d(w, v) \\
& \geq g\left(p_{k}\right)+\sum_{i=k}^{n-1} d\left(p_{i}, p_{i+1}\right)+d(w, v)
\end{aligned}
$$

The latest inequality is due to the invariant itself. The above relation implies that $\left(p_{k}, p_{k+1}, \ldots, w=p_{n}, v=p_{k}\right)$ would be a negative cycle. However, we excluded negative cycles from our graphs under investigation. We conclude that a path associated with a node $w$ cannot convert into a cycle.

As the number of paths is finite, the number of updates is finite for any node $w$. Consequently, the algorithm has a finite number of iterations. The proof of Invariant 1 (c) 2007 The Author. Journal compilation @ 2007 VVS. 
also shows that $g(s)$ is never updated, which implies that $g(s)=0$ is an invariant. Now, we show that $g(w)$ provides us with the shortest path length on termination. As $g(w)$ equals the length of a path by Invariant $1, g(w) \geq \hat{d}(s, w)$. Every edge $(u, v)$ on any path satisfies $d(u, v) \geq g(v)-g(u)$ and hence, every path $P$ from $s$ to $w$ has the property: length $(P) \geq g(w)-g(s)=g(w)$. In conclusion, $g(w)=\hat{d}(s, w)$. Apart from the path in Invariant 1 we can identify a second path, whose definition is based on the following invariant.

InVARIANt 2. For any edge $(u, v) \in E$ with $u=\operatorname{pred}(v), g(v) \geq g(u)+d(u, v)$.

The proof of this invariant is almost trivial. Using this invariant we can prove, analogously to Invariant 1 , that any node $w$ with finite label is associated with a path $\left(s=p_{0}, p_{1}, \ldots, p_{k}=w\right)$ such that $p_{k-1}=\operatorname{pred}\left(p_{k}\right)$. This path is called the predecessor path of $w$. The predecessor path $P$ of a node $w$ satisfies: $g(w) \geq \operatorname{length}(P)$, an immediate result of Invariant 2 and the invariant $g(s)=0$. If strict inequality length $(P)>g(w)$ holds, the predecessor path $P$ differs from the path mentioned in Invariant 1. The discussion in the rest of this paper prefers the use of the predecessor path. On termination of the algorithm, both types of paths provide us with the shortest path.

\subsection{Refining the generic algorithm}

Algorithm 2, a refined version of the generic algorithm, is called the $S$-set algorithm because of the crucial role of the set $S$. The edges adjacent to one node are selected jointly for relaxation. The variable $c$ is an iteration counter, to be used in section 4 .

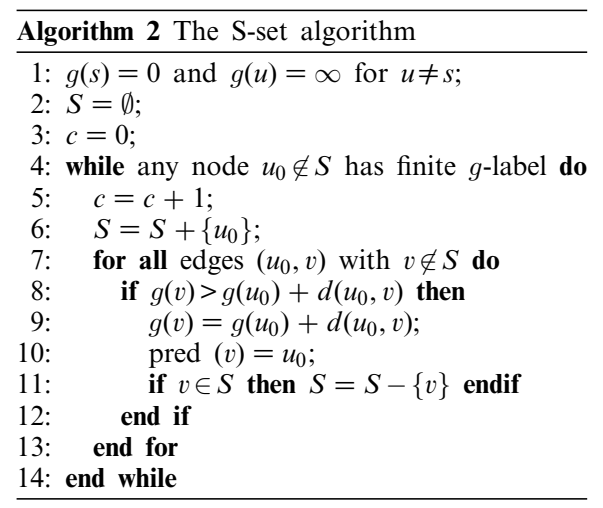

There is an important invariant, the proof of which is left to the reader.

InVARIANT 3. For any $(u, v) \in E$ with $u \in S, g(v) \leq g(u)+d(u, v)$. 
This invariant makes sure that the stop criterion of the $S$-set algorithm implies the stop criterion of the generic algorithm. The $S$-set algorithm is non-deterministic, because the choice of $u_{0}$ is not prescribed. It can be viewed as a family of algorithms, comprising almost all shortest path algorithms. However, one well-known algorithm viz. Bellman-Ford does not belong to this family, but in the case that the data structure for the S-set is a queue, Algorithm 2 is a Bellman-Ford instance.

A great deal of papers, e.g. those of Cherkassky, Goldberg and Radzik (1996) and Gallo and Pallottino (1986), have been devoted to the question as to which data structure of the $S$-set is suitable for which type of input graph. In the current paper this issue is not considered.

\subsection{Using a heuristic estimate}

In some situations one may assume the availability of a so-called heuristic estimate. A heuristic estimate $h$ is defined as a function from $V$ into $\mathbb{R}$. Many search problems, e.g. the traveling salesman problem (TSP) or the 13-14-15 puzzle, can be modelled as a shortest path problem with a heuristic estimate. The value $h(v)$ for any $v \in V$ is chosen such that this value estimates the distance from $v$ to a target node. If no estimation method is available, $h \equiv 0$ is applied.

The $h$-value is a second label for each node next to the $g$-label. The value $g(v)+h(v)$ is referred to as $f(v)$. Using the heuristic estimate, we can define a more specific selection rule for the S-set algorithm. To that end, in front of line 5 or 6 we insert:

choose $u_{0}$ such that $f\left(u_{0}\right)$ is minimal outside $S$.

The $S$-set algorithm with this enhancement is called the heuristic estimate (HE) algorithm. The goal of the heuristic is to narrow the search space, which is defined as the set of nodes with finite $g$-label. Notice that adding a constant $C$ to the function $h$ does not affect the way a run of the HE algorithm proceeds.

\section{Heuristic estimates}

In this section, we address several types of estimates. In section 3.1 a framework is discussed comprising amongst others Dijkstra, $\mathrm{A}^{*}$ and the modified label correcting version as mentioned in Ahuja, Magnanti and OrLin (1989). Section 3.3 treats the so-called non-misleading estimates.

\subsection{Admissible and consistent estimates}

The heuristic estimate $h$ is called admissible with respect to a set $A \subseteq V$, if $h(v)-$ $h(a) \leq \hat{d}(v, a)$ for any $v \in V$ and $a \in A$. We call a heuristic estimate $h$ consistent, if $h$ is admissible with respect to the full node set $V$, or put another way $h(u)-h(v) \leq \hat{d}(u, v)$ (c) 2007 The Author. Journal compilation (c) 2007 VVS. 
for any two nodes $u, v \in V$. In some literature a different but equivalent definition of consistent is found: $h(u)-h(v) \leq d(u, v)$ for any edge $u, v \in V$. For admissible estimates, we have the following important invariant.

INVARIANT 4. If the heuristic estimate is admissible with respect to a set A, then

(a) $\forall u \in S, \forall v \in \bar{S} \cap A, f(u) \leq \hat{d}(s, v)+h(v) \leq f(v)$;

(b) $\forall u \in S \cap A, g(u)=\hat{d}(s, u)$.

Proof. (a) The right-hand inequality is obvious, because every $g$-label is associated with a path by Invariant 1 . Therefore, we focus on the left-hand inequality. Suppose a node $u_{0}$ is selected for insertion into $S$. Let $v$ be any node in $\bar{S} \cap A$ and let $P$ be the shortest path from $s$ to $v$. The first node outside $S$ on this path is denoted by $p$. The two parts of $P$ between $s$ and $p$ and between $p$ and $v$ are called $P_{1}$ and $P_{2}$ respectively. As a result of Invariant 3 we have length $\left(P_{1}\right) \geq g(p)-$ $g(s)=g(p)$. As $v \in A$, length $\left(P_{2}\right)=\hat{d}(p, v) \geq h(p)-h(v)$. Combining these inequalities gives $\hat{d}(s, v)=$ length $(P) \geq g(p)+h(p)-h(v)$ or equivalently $\hat{d}(s, v)+h(v) \geq g(p)+h(p)$. The latest value is $\geq f\left(u_{0}\right)$ by the selection criterion. Any vertex $v \in S \cap A$ is not removed from $S$, because $g(v)=\hat{d}(s, v)$ by invariant $(b)$ and hence, $g(v)$ has a permanent label. So $\bar{S} \cap A$ is not enhanced. We conclude that the invariant is preserved in every iteration. (b) When a node $v \in \bar{S} \cap A$ is selected, then node $p$ as defined in part (a) coincides with $v$, so $v=p$. In part $(a)$ we have seen $g(p)=\hat{d}(s, p)$.

If the heuristic estimate is consistent, every node is inserted into $S$ exactly once. In that case the algorithm is called a label-setting instance, as every node has a permanent label, when inserted into $S$. The run-time is $O\left(n^{2}\right)$ in that case. If the estimate is not consistent, a node in $S$ may take a new label and leave the $S$-set. Then the algorithm is called label-correcting.

Invariant 4 implies that, when the heuristic estimate algorithm runs on a graph with an admissible estimate, each $f$-value in $\bar{S} \cap A$ is greater than or equal to any $f$-value in $S \cap A$. Consequently, the $f$-values of the nodes of $A$ consecutively selected make up a non-decreasing series. Notice that the heuristic estimate $h \equiv 0$ is consistent, provided that the weights are non-negative. The HE algorithm running on graph with non-negative weights and $h \equiv 0$ is exactly Dijkstra's algorithm.

\subsubsection{The $A^{*}$ algorithm}

Most of the textbooks on AI discuss $\mathrm{A}^{*}$, which is defined in our terminology as the HE algorithm using an estimate admissible with respect to a set $A$ consisting of a single node. Curiously, this algorithm is not well-known among researchers of the OR community, which is focused on Dijkstra and Bellman-Ford. Conversely, the AI literature pays a lot of attention to $\mathrm{A}^{*}$, but ignores widespread algorithms such (c) 2007 The Author. Journal compilation (c) 2007 VVS. 
as Dijkstra and Bellman-Ford. Only recently, some OR researchers working on real road networks included $A *$ in their investigations (e.g. Goldberg and Harrelson, 2005).

The A* algorithm is presented in most of the AI textbooks (e.g. Pearl, 1984; Russell, 2003), as a suitable method to solve search problems, such as the 13-14-15 puzzle. The best-first branch-and-bound solution for TSP using a heuristic lower bound is actually an $\mathrm{A}^{*}$ instance.

\subsection{Running with or without heuristic function}

Given a graph with weight function $d$ and a heuristic estimate $h$, we introduce new weights:

$$
d^{\prime}(u, v)=d(u, v)+h(v)-h(u)
$$

When running the HE algorithm, we define an alternate label $g^{\prime}(u)=f(u)-h(s)$ for any $u$. The initial labels $g(s)$ and $g^{\prime}(s)$ are $=0$. Whenever $g(v)$ is given a new value $g(v)=g(u)+d(u, v)$, this update can also be viewed as setting the alternate label $g^{\prime}(v)$ to $g^{\prime}(u)+d^{\prime}(u, v)$. It follows that the HE algorithm using an $h$-function is equivalent to HE on a graph with transformed weights and a heuristic $\equiv 0$. If the heuristic is consistent, then $d^{\prime} \geq 0$ and the algorithm running on the transformed graph is an instance of Dijkstra's algorithm. A graph with an admissible heuristic corresponds to one with possibly negative weights, but where every distance $\hat{d}(v, a)$ with $v \in V$ and $a \in A$ is non-negative.

\subsection{Non-misleading and proper estimates}

Suppose we focus on one target node $t$. A heuristic estimate $h$ is called non-misleading if any two nodes $m$ and $n$ have the property:

$$
\text { length }(P)+h(m)<\text { length }(Q)+h(n) \Rightarrow \text { length }(P)+\hat{d}(m, t) \leq \text { length }(Q)+\hat{d}(n, t)
$$

for any two paths $P$ and $Q$ from $s$ to $m$ and to $n$ respectively. An example of a nonmisleading estimate is shown in Figure 1. A weaker notion is proper. A heuristic

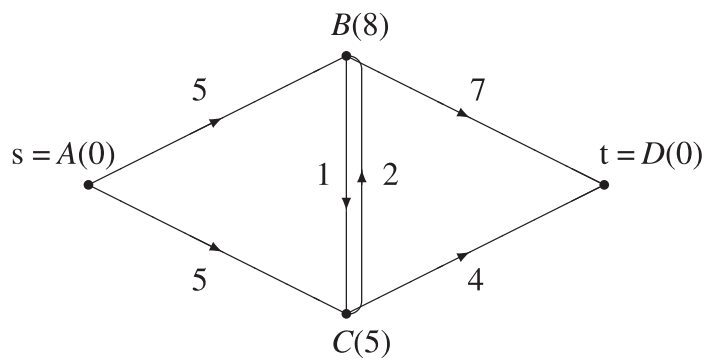

Fig. 1. A non-misleading estimate the $h$-values are in brackets. 
estimate $h$ is called proper if (2) only holds for any two paths $P$ and $Q$, that are not included in each other.

INVARIANT 5. Suppose the HE algorithm runs on a graph with non-negative weights and with a proper estimate, where ties in the selection criterion are solved in favour of the smallest $g$-value.

(a) $\forall u \in S, \forall v \notin S, g(u)+\hat{d}(u, t) \leq g(v)+\hat{d}(v, t) \vee g(u) \leq g(v)$;

(b) $\forall u \in S, g(u)=\hat{d}(s, u)$.

Proof. (a) When a node $u_{0}$ is selected, the selection criterion implies that:

$$
\forall v \notin S, g\left(u_{0}\right)+h\left(u_{0}\right)<g(v)+h(v) \vee g\left(u_{0}\right) \leq g(v) .
$$

Because of invariant (b), no node has been deleted from $S$ and hence, all predecessors are in $S$. Consequently, the predecessor paths of $u_{0}$ and $v$ cannot be subpaths of each other. As a result of the Invariants 2 and 3, the lengths of both predecessor paths are equal to the corresponding $g$-labels. As the estimate function $h$ is proper, we state for all $v \notin S$ :

$$
g\left(u_{0}\right)+h\left(u_{0}\right)<g(v)+h(v) \Rightarrow g\left(u_{0}\right)+\hat{d}\left(u_{0}, t\right) \leq g(v)+\hat{d}(v, t) .
$$

If $g(v)$ is not changed, invariant (a) holds for $v$ and any $u \in S$. If $v$ is given a new label $g(v)=g\left(u_{0}\right)+d\left(u_{0}, v\right)$, then for any $u \in S$ either $g(u) \leq g\left(u_{0}\right) \leq g(v)$ or:

$$
g(u)+\hat{d}(u, t) \leq g\left(u_{0}\right)+\hat{d}\left(u_{0}, t\right)=g(v)-d\left(u_{0}, v\right)+\hat{d}\left(u_{0}, t\right) \leq g(v)+\hat{d}(v, t),
$$

where the rightmost inequality is a consequence of a general inequality for the $\hat{d}$-function.

(b) Let $P$ be the shortest path from $s$ to $u_{0}$. Similar to the proof of Invariant 4 we derive: length $\left(P_{1}\right) \geq g(p)$. If $g\left(u_{0}\right)+\hat{d}\left(u_{0}, t\right) \leq g(p)+\hat{d}(p, t)$ in (a) holds, then using length $\left(P_{2}\right)=\hat{d}\left(p, u_{0}\right) \geq \hat{d}(p, t)-\hat{d}\left(u_{0}, t\right)$ we obtain: length $(P) \geq g(p)+\hat{d}(p, t)-$ $\hat{d}\left(u_{0}, t\right) \geq g\left(u_{0}\right)$. If $g\left(u_{0}\right) \leq g(p)$ in (a) holds, then length $(P) \geq \operatorname{length}\left(P_{1}\right) \geq g(p) \geq g\left(u_{0}\right)$.

This invariant shows that the HE algorithm running with a proper estimate is a label-setting instance. In contrast with a consistent estimate, the $f$-values successively selected do not generate an increasing series.

The notion of non-misleading has been introduced in IBARAKI (1977) in the context of the branch-and-bound method. The definition in that paper differs from the current one, which is taken from BAGCHI and MAHANTI (1983). They are equivalent in case of an acyclic graph. The notion proper has been introduced in BAGCHI and MAHANTI (1983). In that paper, a weaker version of Invariant 5 was also presented. The result that the label-setting version is applicable to a proper heuristic estimate, is new. 


\section{An application of binary numbers}

In this section, we present a pathological graph, where the HE algorithm has an exponential run time of $2^{|V|-1}$. Consider the following graph $K$ where the nodes are denoted by integers.

$$
\begin{aligned}
V & =\{1,2, \ldots, n, n+1\} \\
E & =\{(u, v) \mid u, v \in V \text { and } u>v\} \\
d(u, v) & =2^{u-1}-2^{v} \\
s & =n+1
\end{aligned}
$$

Suppose we insert the following statement into the $S$-set algorithm before line 5 or 6 :

choose $u_{0}$ such that $u_{0}$ is the smallest integer outside $S$.

Later on in this section, we show that the $S$-set algorithm with this selection rule is an instance of HE. Running the $S$-set algorithm on graph $K$ with this selection rule provides an interesting application of the binary number system, expressed by Invariant 7 . This invariant deals with the variable $c$, the iteration counter (see line 5 in the $S$-set algorithm). The following definition $I(u)$ is useful for Invariant 6 : $I(v)=\{p \mid v<p \leq n$ and $p$ integer $\}$.

INVARIANT 6. Let $I(v) \cap S$ for any $v$ be given by $\left\{p_{1}, \ldots, p_{k}\right\}$ with $p_{1}>p_{2}>\cdots>p_{k}$. Then the predecessor path of $v$ is $\left(n+1=p_{0}, p_{1}, p_{2}, \ldots, p_{k}, v\right)$ and $g(v)$ equals the length of this path.

Proof. Suppose $u_{0}$ is selected to be inserted into $S$. Let the set $I\left(u_{0}\right) \cap S$ be given by $\left\{p_{1}, \ldots, p_{h}\right\}$. By the invariant itself the predecessor path of $u_{0}$ is $\left(s=p_{0}, p_{1}, p_{2} \ldots\right.$, $\left.p_{h}, u_{0}\right)$ and this path has length $=g\left(u_{0}\right)$. As $u_{0}$ is the smallest integer outside $S$ according to the selection rule, the set $\left\{u_{0}-1, u_{0}-2, \ldots, 2,1\right\}$ is also included in $S$.

When $u_{0}$ is inserted into $S$, any node $v$ with $v>u_{0}$ is not affected, because every edge goes from a higher to a lower number. So we consider only nodes $v$ with $u_{0}>v \geq 1$. For such a node $v$, the predecessor path has the form $\left(n+1=p_{0}, p_{1}, p_{2} \ldots\right.$, $\left.p_{h}, u_{0}-1, u_{0}-2, \ldots, v\right)$. Notice that every edge in the sequence $\left(u_{0}-1, u_{0}-2, \ldots, v\right)$ has weight equal to 0 . As the length of this path equals $g(v)$ by the invariant, we have:

$$
\begin{aligned}
g(v) & =\sum_{i=0}^{i=h-1} d\left(p_{i}, p_{i+1}\right)+d\left(p_{h}, u_{0}-1\right) \\
& =\sum_{i=0}^{i=h-1} d\left(p_{i}, p_{i+1}\right)+2^{p_{h}-1}-2^{u_{0}-1} .
\end{aligned}
$$

The edge $\left(u_{0}, v\right)$ is relaxed, because 


$$
\begin{aligned}
g\left(u_{0}\right)+d\left(u_{0}, v\right) & =\sum_{i=0}^{i=h-1} d\left(p_{i}, p_{i+1}\right)+d\left(p_{h}, u_{0}\right)+d\left(u_{0}, v\right) \\
& =\sum_{i=0}^{i=h-1} d\left(p_{i}, p_{i+1}\right)+2^{p_{h}-1}-2^{u_{0}}+2^{u_{0}-1}-2^{v} \\
& =\sum_{i=0}^{i=h-1} d\left(p_{i}, p_{i+1}\right)+2^{p_{h}-1}-2^{u_{0}-1}-2^{v} \\
& =g(v)-2^{v}
\end{aligned}
$$

Hence, when $u_{0}$ is inserted into $S$, every $v$ with $u_{0}>v \geq 1$ takes a new $g$-label and leaves the $S$-set. For each $v$ in this range the new set $I(v) \cap S$ is equal to $\left(p_{1}, \ldots\right.$, $\left.p_{h}, u_{0}\right)$. Because $\left(u_{0}, v\right)$ is relaxed, the new predecessor path of $v$ consists of the predecessor path of $u_{0}$ enhanced with $\left(u_{0}, v\right)$. So this path conforms to the new value of $I(v) \cap S$. As a result of Invariants 2 and 3 the length of the predecessor path equals $g(v)$.

INVARIANT 7. Let the binary notation of $c-1$ be given by $a_{n} a_{n-1} \ldots a_{2} a_{1}$. This notation and the set $S$ are related by the equality: $S \backslash\{s\}=\left\{p \mid a_{p}=1\right\}$

Proof. Notice that the invariant holds when $c=1$ (only source $n+1$ has been inserted). Assume that the invariant holds at the time $u_{0}$ is selected, so $c-1$ has the form: $a_{n} a_{n-1} \ldots a_{u_{0}+1} 01 \ldots 1$. In the next iteration, $u_{0}$ enters $S$ causing the nodes $v$ with $1 \leq v<u_{0}$ to leave the $S$-set, as we have seen in the proof of Invariant 6 . Adding 1 to the old $(c-1)$-value gives $a_{n} a_{n-1} \ldots a_{u_{0}+1} 10 \ldots 0$, which is in accordance with the new $S$-set.

Now it is obvious that the number of iterations equals exactly $2^{n}$, because $c-1=2^{n}-$ 1 , when $S=V$ is achieved ( $c-1$ consists of $n$ digits 1$)$. Invariant 6 implies that $g(v)$ is a multiple of $2^{v}$ at any time, because the length of the predecessor path is composed of numbers $2^{p}$ with $p \geq v$. Every subset of $I(v)$ is equal to $I(v) \cap S$ at some time. Consequently, $g(v)$ takes $2^{|I(v)|}=2^{n-v}$ different values, ranging from $0 * 2^{v}$ up to $\left(2^{n-v}-1\right) * 2^{v}=2^{n}-2^{v}$. The proof of Invariant 6 also shows that, whenever $v$ is given a new label, the label is decreased by $2^{v}$.

The following nodes are inserted successively after node $s: 1,2,1,3,1,2,1,4,1$, $2,1,3,1,2,1, \ldots$ This series is also known from 'Towers of Hanoi', a famous topic in recreational mathematics, which is often treated in textbooks on programming to illustrate recursion.

The definition of graph $K$ has been presented in PIJLs (1991) and in a slightly different form in AHUJA et al. (1989). It is based upon an earlier instance, introduced in Martelli (1977). 
By adding target node 0 and adding an edge $(1,0)$ with $d(1,0)=2^{n}$ to the graph $K$, we obtain an instance $K^{\prime}$ of the $\mathrm{A}^{*}$-algorithm. It follows that also $\mathrm{A}^{*}$ may have exponential run time. The following lemma implies that the above instance $K$ with $2^{|V|-1}$ iterations achieves a maximal number of iterations.

Lemma 1. Consider two arbitrary iterations of the S-set algorithm. Then there is a node $u$ with $u \notin S$ after the earlier iteration and $u \in S$ after the later iteration.

Proof. Let $g_{1}$ and $g_{2}$ denote the $g$-labels after the two iterations under consideration. Let $p$ be a node such that $g_{2}(p)<g_{1}(p)$. Let the predecessor path after the second iteration be given by $\left(s=p_{0}, p_{1}, \ldots, p_{n}=p\right)$. Let $p_{k-1}$ and $p_{k}$ be two consecutive nodes in this path, such that $g_{1}\left(p_{k-1}\right)=g_{2}\left(p_{k-1}\right)$ and $g_{1}\left(p_{k}\right)>g_{2}\left(p_{k}\right)$. Such nodes must exist, because of the fact that $g_{1}\left(p_{0}\right)=g_{2}\left(p_{0}\right)$ and $g_{1}\left(p_{n}\right)>g_{2}\left(p_{n}\right)$. We conclude that the latest update of $g\left(p_{k}\right)$ has been executed more recently than the latest update of $g\left(p_{k-1}\right)$. Hence, $p_{k-1}$ has not been updated after it became $p_{k}$ 's predecessor, so $u=p_{k-1}$ is in $S$ after the second iteration. The invariants 2 and 3 imply that $g_{2}\left(p_{k}\right)=g_{2}\left(p_{k-1}\right)+d\left(p_{k-1}, p_{k}\right)$. As $g_{1}\left(p_{k}\right)>g_{2}\left(p_{k}\right)=g_{2}\left(p_{k-1}\right)+d\left(p_{k-1}, p_{k}\right)=g_{1}\left(p_{k-1}\right)+$ $d\left(p_{k-1}, p_{k}\right)$, we conclude from Invariant 3 that $u=p_{k-1}$ was not in $S$ after the first iteration.

By Lemma 1 the $S$-set after each iteration is unique. In the very first iteration of the algorithm $s$ is inserted into $S$ and this node remains in $S$ throughout the algorithm. Hence, the number of iterations is at most the number of subsets (the empty set included) of $V \backslash\{s\}$, which number equals $2^{|V|-1}$. We see that graph $K$ having $2^{n}=2^{|V|-1}$ iterations attains the maximal number of iterations.

I conclude this section with a personal note. When I communicated my discovery on the role of binary numbers to Antoon, he responded: 'Wat is wiskunde toch mooi!' (How beautiful mathematics is!) No doubt, we agree with this statement.

\section{Real road networks and bidirectional search}

The past few years have witnessed a revival of shortest path algorithms, in particular, algorithms focused on real road networks. The instance that is solved by the present-day car navigators, is the single-source single-target version of the shortest path problem. Bidirectional search is a powerful method for finding the shortest path in a road network from one point to another and is applied therefore in almost any navigator. The method originates from PoHL (1971). Substantial improvements were proposed by, amongst others, IKEDA et al. (1994), GoldBerg and HARRELSON (2005) or YAMAGUCHI and MASUdA (2006). Given a source and a target, bidirectional search works as follows. The HE algorithm runs simultaneously on two sides, starting from the source and the target, respectively, so we have forward and backward search. The (c) 2007 The Author. Journal compilation (c) 2007 VVS. 
backward search uses different edges: just edges $(v, u)$ such that $(u, v)$ in the original set $E$, are used. The cost of an edge $(v, u)$ equals the value of the original edge $(u, v)$. Each search process has its own heuristic. The algorithm stops when the $S$-sets of the two searches have some overlap. The exact termination criterion depends on the properties of the heuristics. (An detailed treatment of bidirectional search can be found in PIJLs and Post, 2006.)

\subsection{Suitable estimates}

An obvious consistent heuristic in road networks is: $h(v)$ equal to the astronomical distance from $v$ to $t$. HE running with this heuristic estimate visits considerably less nodes than Dijkstra's algorithm $(h \equiv 0)$ as is illustrated by some pictures in Roos (2004) or in KLunder and Post (2006). In Goldberg and Harrelson (2005) a new estimate was introduced, incorporated into the so-called ALT algorithm, which stands for $\mathrm{A}^{*}$, Landmarks and Triangle inequality. The algorithm needs some preprocessing of the road network. A number of nodes are designated as landmarks. In advance, $\hat{d}(v, m)$ is computed for any node $v$ and every landmark $m$. Using the triangle inequality we can show that the choice $h(v)=\hat{d}(v, m)-\hat{d}(t, m), m$ being a fixed landmark, gives a consistent heuristic. If we choose $m$ close to $t$, a tight lower bound to $\hat{d}(v, t)$ is obtained. (An extensive discussion is given in GoldBERG and HARrelson, 2005.)

\subsection{New methods}

Apart from the landmarks, several other new heuristics have been invented in the past few years. All of them need an elaborate preprocessing phase to compute the heuristic. (Examples of new heuristics can be found in MöHring et al., 2005; WAGNer, Willhalm and Zaroliagis, 2005; or Sanders and Schultes, 2005.)

\section{The search space}

In this section we want to find which set of nodes is visited during the HE algorithm. For an admissible $h$ the set $R_{h}(H)$ with $H$ a real number is defined as the set of nodes $v$ for which a path $P=\left(s=p_{0}, p_{1}, \ldots, p_{n}=v\right)$ exists such that for $0 \leq k \leq n$,

$$
\sum_{j=0}^{k-1} d\left(p_{j}, p_{j+1}\right)+h\left(p_{k}\right) \leq H,
$$

If the heuristic $h$ is consistent, the definition can be simplified. The inequality (4) holds automatically for $1 \leq k<n$ if it holds for $k=n$.

The following proposition follows immediately from the definition: $h_{1} \leq h_{2} \wedge H_{1} \geq$ $H_{2} \Rightarrow R_{h_{2}}\left(H_{2}\right) \subseteq R_{h_{1}}\left(H_{1}\right)$. 
During execution of the $S$-set algorithm the variable $F$ is defined at any time as:

$$
F=\min \{f(v) \mid v \in S\}
$$

Invariant 4 shows that, in case of a consistent estimate, $F \leq f(v)$ for any $v \notin S$. If the estimate is not consistent, for some $v \notin S$ the inequality $f(v)<F$ may happen. We introduce the following selection criterion into the $S$-set algorithm:

(1) select, if any, an arbitrary node $u_{0} \notin S$ with $f\left(u_{0}\right) \leq F$

(2) if $f(u)>F$ for any $u \notin S$, select $u_{0}$ such that $f\left(u_{0}\right)$ is minimal outside $S$ (and after inserting $u_{0}$ into $S, f\left(u_{0}\right)$ is the new $F$-value)

We call the $S$-set algorithm with this additional selection rule the F-algorithm. Notice that the selection criterion in section 2.3 is a special case of this one. If the estimate is consistent, the two criteria are equivalent. As soon as $t$ is inserted into $S$, the algorithm can stop, since $g(t)=\hat{d}(s, t)$ because of Invariant 4 .

The following property holds during execution: $S \subseteq R_{h}(F)$ and on termination: $S \subseteq R_{h}(\hat{d}(s, t))$. At the moment that every node $v \notin S$ satisfies $f(v)>F$, then even $S=R(h, F)$.

Although $H$ may be any real number, there is finite number of distinct sets $R_{h}(H)$ for a given estimate $h$ on a graph $G$. To be more precise, there is a finite series of values $H_{0}, H_{1}, H_{2}, \ldots, H_{n}$ with the property that any set $R_{h}(H), H$ a real number, is equal to $R_{h}\left(H_{k_{0}}\right)$ with $k_{0}$ in the range $0, \ldots, n$. Consequently, the series of values assigned to the variable $F$ during execution of the $F$-algorithm is equal to the series $H_{0},=0, H_{1}, H_{2}, \ldots, H_{n}$. So the $F$-algorithm may be viewed as a method to trace the sets $R_{h}\left(H_{k}\right), 0 \leq k \leq n$. The IDA algorithm (see PEARL, 1984; Russell, 2003) is based upon this principle. In the IDA* algorithm, the sets $R_{h}\left(H_{k}\right), 0 \leq k \leq n$ are traced successively using backtracking. This makes the algorithm appropriate to be used in restricted memory.

\section{Concluding remarks}

According to Antoon there is no need to sum up and repeat the results in a conclusion at the end of a paper. my conclusion is different. I remember Antoon as my supervisor and master with a strong commitment to his students. In memory of him and in great gratitude I have written this article.

\section{References}

Ahuja, R. K., T. L. Magnanti and J. B. OrLin (1989), Network flows, theory, algorithms and applications, Prentice Hall, Englewood Cliffs, New Jersey.

BagChi, A. and A. Mahanti (1983), Search algorithms under different kinds of heuristics - a comparative study, Journal American Computing Machinery 30, 1-21.

Cherkassky, B. V., A. V. Goldberg and T. Radzik (1996), Shortest path algorithms: theory and experimental evaluation, Mathematical Programming 73, 129-174. 
Gallo, G. and S. Pallottino (1986), Shortest path methods: a unifying approach, Mathematical Programming Study 26, 38-64.

Garey, M. R. and D. S. Johnson (1979), Computers and intractability, A guide to the theory of NP-completeness, Freeman, New York.

Goldberg, A. V. and C. Harrelson (2005), Computing the shortest path: A* search meets graph theory, in: 16th Annual ACM-SIAM Symposium on Discrete Algorithms (SODA'05), extended version. Technical Report MSR-TR-2004-24, (2004), Microsoft Research.

Goodrich, M. T. and R. Tamasia (2002), Algorithm design, John Wiley and Sons, New York.

IBARAKI, T. (1977), The power of dominance relations in branch-and-bound algorithms, Journal American Computing Machinery 24, 264-279.

Ikeda T. K., M.-Y. Hsu, H. Inai, S. Nishimura, H. Shimoura, T. Hashimoto, K. Tenmoku, and K. Мгтон (1994), A fast algorithm for finding better routes by AI search techniques, in: Proceedings Vehicle Navigation and Information Systems Conference, Yokohama, IEEE, 291-296.

KLunder, G. A. and H. N. Post (2006), The shortest path problem on large scale real road networks, Networks 48, 184-192.

Martelli, A. (1977), On the complexity of admissible search algorithms, Artificial Intelligence 8, 1-13.

Möhring R. H., H. Schilling, B. Schütz, D. Wagner and T. Willhalm (2005), Partitioning graphs to speed up Dijkstra's algorithm, in: S. E. Nikoletseas (ed.), Proceedings Experimental and Efficient Algorithms: 4th International Workshop, Lecture Notes in Computer Science 3503, Springer, 198-202.

Papadimitriou, Chr. and K. Steiglitz (1982), Combinatorial optimization: algorithms and complexity, Prentice Hall, Englewood Cliffs, New Jersey.

Pearl, J. (1984), Heuristics, intelligent search strategies for computer problem solving, Addison Wesley, London.

PoHL, I. (1971), Bi-directional search, Machine Intelligence 6, 124-140.

Pisls, W. (1991), Shortest paths and game trees, PhD thesis, Erasmus University Rotterdam, Rotterdam.

PiJls, W. and H. N. Post (2006), Some theoretical and practical aspects of Bidirectional Search, Econometric Institute Report 2006-41, Erasmus University Rotterdam.

Roos, C. (2004), De tijd zal het leren, inaugural address (in Dutch), Nieuw Archief voor Wiskunde 5, 186-202.

Russell, S. J. and P. Norvig (2003), Artificial intelligence, a modern approach, Prentice Hall, Englewood Cliffs, New Jersey.

Sanders, P. and D. Schultes (2005), Highway hierarchies hasten exact shortest path queries, in: G. S. Brodal and S. Leonardi (eds), Proceedings 17th European Symposium on Algorithms (ESA), Lecture Notes in Computer Science 3669, Springer, 568-579.

Wagner, D., T. Willhalm and Chr. Zaroliagis (2005), Geometric containers for efficient shortest-path computation, ACM Journal of Experimental Algorithms 10, 1-30.

YAmAGUCHI K. and S. Masuda (2006), An A* algorithm with a new heuristic distance function for the 2-terminal shortest path problem, IEICE Transactions on Fundamentals of Electronics E89-A, 544-550.

Received: February 2006. Revised: August 2006. 